\section{A case of Churg-Strauss syndrome complicated with bilateral central retinal artery occlusion}

\author{
Ken Uchibori, Emi Shinohara, Haruhiko \\ Furusawa, Shinichiro Tominaga, Ichiro \\ Natsume, Minoru Ohkouchi \\ Department of Pulmonology, Yokosuka \\ Kyosai Hospital, Yokosuka, Japan
}

\begin{abstract}
A 56-year-old Japanese woman treated for bronchial asthma came to our hospital because of sudden vision loss of the left eye and plantar numbness of the right foot. Central retinal artery occlusion (CRAO) was diagnosed. Vision loss of the right eye also appeared, and the numbness of the right foot worsened. She was diagnosed with Churg-Strauss syndrome (CSS) due to bronchial asthma, eosinophilia, and vasculitis (CRAO and numbness). Her symptoms were unresponsive to systemic corticosteroid therapy, but improved when cyclophosphamide pulse was added. Vision disorders are uncommon manifestations of CSS. Close co-ordination with ophthalmologists is important for diagnosis and treatment.
\end{abstract}

\section{Introduction}

Ophthalmic complications in Churg-Strauss syndrome (CSS) have a reported incidence of $16 \% .{ }^{1,2}$ We experienced a case who developed bilateral central retinal artery occlusion (CRAO) within a short period.

\section{Case Report}

The patient was a 56-year-old woman employed as an office clerk. She had been diagnosed with bronchial asthma at the age of 42 and was receiving two daily doses of fluticasone propionate $(500 \mu \mathrm{g}) /$ salmeterol $(50 \mu \mathrm{g})$ inhalation powder and two daily doses of theophylline $(200 \mu g)$. One of her children also had bronchial asthma. She had a smoking history of 22 years (a pack a day from the age of 28 to 50), social drinking, and had no history of breeding pets.

In 1995, she had been diagnosed with bronchial asthma after suffering an episode of wheezing that recovered with treatment. Her family history and her reaction to house dust supported the diagnosis. She began treatment with the above-mentioned drugs from 2004, with no use of the leukotriene antagonists, and suffered asthma attacks requiring systemic corticosteroids two to three times a year up to now. At the end of May 2009, she experienced numbness of the right plantar for the first time. The symptom was initially evaluated as lumbar spondylosis not requiring orthopedic treatment, but it persisted without improvement. At the beginning of July she suddenly lost vision of the left eye. Her left corrected eyesight was 20/200. Her ophthalmologist diagnosed occlusion of the left central retinal artery and referred her to the ophthalmologist in our hospital. A cherry red spot and retinal whitening appeared on funduscopy. Retinal reperfusion was confirmed to be in normal range based on a normal arm-to-retina circulation time in fluorescent dye angiography (22 s). She was discharged from the hospital not long after admission, as her left corrected eyesight recovered from $20 / 300$ to $20 / 60$ in response to urokinase and several other agents. A week later, however, she was readmitted because of a sudden loss of right vision. Corrected vision of the right eye was assessed as 20/2000 and funduscopy revealed a cherry red spot of the same type observed earlier in the left eye (Figure 1). Occlusion of the right central retinal artery was diagnosed based on a prolonged arm-to-retina circulation time of more than $1 \mathrm{~min}$ in fluorescent dye angiography, and treatment was started (Figure 2). Persistent numbness of lower limbs, a slight fever just over $37^{\circ} \mathrm{C}$, and an elevated white blood cell (WBC) count of $34,000 / \mu \mathrm{L}$ (with eosinophil elevation up to $84.5 \%$ ) were noted at readmission. She was therefore referred to our department for both further inspection and asthma management at the end of July.

On admission, she had a body temperature of $37.5^{\circ} \mathrm{C}$, blood pressure of $108 / 55 \mathrm{mmHg}$, and heart rate of 78 beats/min. Slight damp rales were audible in bilateral lung fields, but no evident wheezing was noted. None of the other findings were abnormal. Neurologic examination revealed sensory disturbance with numbness of the sole of the right foot and a slightly weak knee jerk reflex on both sides, but no muscle weakness was noted. Her right visual field was lost on the nasal side without loss of hand motion visual acuity. Her left visual field was stained with a purple spot that obstructed her visual field but posed no significant loss of visual field or visual acuity. Her corrected visual acuity was 20/1000 on the right and 20/30 on the left. A dynamic vision field examination revealed a central scotoma in the right visual field extending 40 degrees horizontally from the center of the vision field to the nasal side and 120 degrees in the vertical direction. Two scotomas were identified in the left visual field, i.e. a central scotoma and a paracentral scotoma in the nasal field. The laboratory data showed significant increases of WBCs $(34,000 / \mu \mathrm{L})$ and eosinophils $(28,730 / \mu \mathrm{L})$, elevated C-reactive
Correspondence: Ken Uchibori, Department of Pulmonology, Yokosuka Kyosai Hospital, 1-16 Yonegahamadori, 238-8558 Yokosuka, Japan. Tel. +81.046.822.2710 - Fax: +81.78.929.2387.

E-mail: k-uchibori@hp.pref.hyogo.jp

Key words: Churg-Strauss syndrome, allergic granulomatosis and angiitis, central retinal artery occlusion, eosinophilia, multiple mononeuropathy.

Contributions: the authors contributed equally.

Conflict of interests: the authors declare no potential conflict of interests.

Received for publication: 5 December 2012. Revision received: 11 May 2013.

Accepted for publication: 24 May 2013.

This work is licensed under a Creative Commons Attribution NonCommercial 3.0 License (CC BYNC 3.0).

(C) Copyright Ken Uchibori et al., 2013

Licensee PAGEPress, Italy

Chest Disease Reports 2013; 3:e1

doi:10.4081/cdr.2013.e1

protein and lactate dehydrogenase (790 U/L), and an elevated sedimentation score. The patient was negative for both myeloperoxidaseantineutrophil cytoplasmic antibody (ANCA) and proteinase-3-ANCA autoantibodies. A chest computed tomography (CT) scan revealed peribronchial small vesicular shadows, patchy ground-glass opacities, and thickening of the bronchus wall (Figure 3). Nerve conduction studies revealed axonopathy with laterality consistent with multiple mononeuropathy; left dominant motor disorder of the tibial nerve [decreased compound muscle action potential amplitude (CMAP amp) of $3.19 \mathrm{mV}$ and normal motor nerve conduction velocity (MCV) of 52.6 $\mathrm{m} / \mathrm{s}]$; sensory disorder of the right medial plantar nerve [decreased sensory nerve action potential amplitude (SNAP amp) of $2.1 \mu \mathrm{V}$ and normal sensory nerve conduction velocity (SCV) of $50.4 \mathrm{~m} / \mathrm{s}$ ]; and no disorders of the fibula nerve or other nerves. Carotid echography, ankle brachial pressure index, and head magnetic resonance imaging angiography were normal. Echocardiography showed a normal ejection fraction of $60 \%$ and mild hypokinesis in part of the septal wall. There were no pathological findings in the gastric mucosal biopsy. The CRAO was not linked to any evidence of underlying heart disease or arteriosclerosis. Instead, the CRAO and multiple mononeuropathy were evaluated as symptoms of vasculitis. The patient was diagnosed with CSS based on clinical manifestations consistent with the triad of CSS: preceding bronchial asthma, prominent elevated eosinophils, and evidence of vasculitis. Ophthalmologic treatments alone failed to 
improve the patient's visual field or eyesight to a satisfactory level. Similarly, poor effects were obtained by methyl-prednisolone (mPSL) $1 \mathrm{~g}$ pulse therapy administered for 3 days at the end of June (Figure 4) followed by systemic corticosteroid [daily prednisolone (PSL) of 1 $\mathrm{mg} / \mathrm{kg}$ ]. Her eyesight remained unimproved, the numbness of the sole of the left foot and bilateral numbness worsened, and neuropathy deteriorated in the nerve conduction study. Cyclophosphamide (CPA) pulse was administered at a dose of $500 \mathrm{mg} / \mathrm{m}^{2}$ and her condition stabilized after the second pulse. The PSL was tapered to a dose of $25 \mathrm{mg} /$ day and the patient was discharged. The PSL was gradually tapered to $10 \mathrm{mg} /$ day and CPA pulse was administered in parallel once every 4 weeks up to May 2010 (10 pulses in total). Her visual field and eyesight recovered to a level sufficient not to interfere with daily life, but her inability to distinguish numbers and numbness of the lower limbs persisted without improvement. Bronchial asthma remained stable during the whole period and the small vesicular shadow found in chest-CT scan vanished 1 month after the start of treatment (Figure 5). The eponym of Churg-Strauss syndrome was replaced by eosinophilic granulomatosis with polyangiitis according to the 2012 revised international Chapel Hill Consensus Conference (CHCC2012) nomenclature of vasculitides.

\section{Discussion}

When Churg and Strauss reported CSS in 1951 they described clinical and pathological discrepancies that distinguished the disease from traditional polyarteritis nodosa. ${ }^{3}$ The current defi- nition of CSS is based on the classification by the American College of Rheumatology. ${ }^{4}$ The typical clinical course is the development of systemic vasculitis with prominent elevation of eosinophils preceded by several years of bronchial asthma or allergic rhinitis. ${ }^{5}$ Though pathologic findings were absent in our patient, we evaluated her case as typical CSS based on initial symptoms of plantar numbness, sudden vision loss, visual field disorder, and fever onset during bronchial asthma treatment. The chest CT scan revealed vesicular shadows recognizable as bronchiole lesions in peripheral lung fields. These lesions were inconsistent with the findings reported earlier, but their extinction after CSS treatment implicated them as a complication of CSS. While not prevalent as complications of CSS, eye manifestations have a reported incidence of about $16 \%$ in CSS patients. Ischemic lesions caused by necrotic

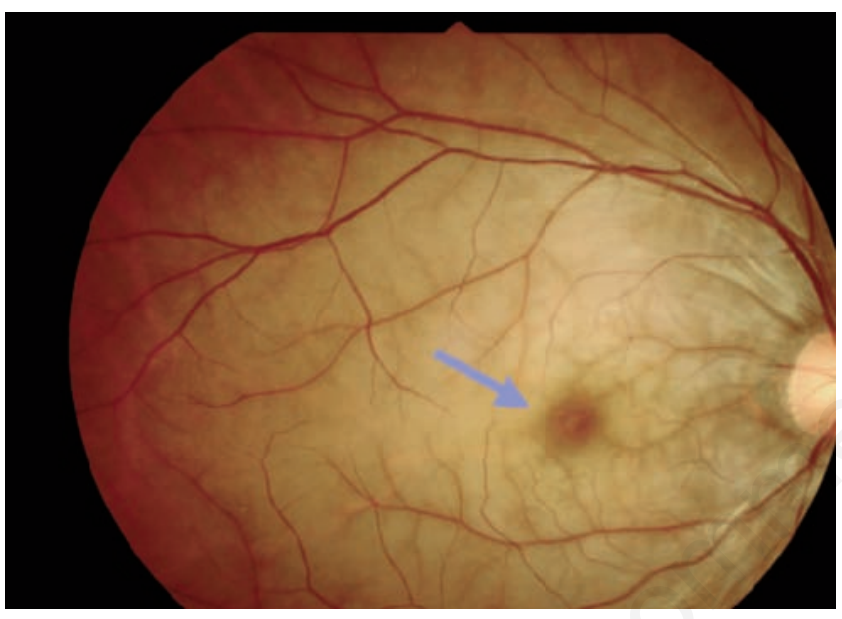

Figure 1. Examination of the right ocular fundus at the onset of right central retinal artery occlusion. Retinal whitening and a cherry red spot (indicated by the arrow) were observed (right retina).

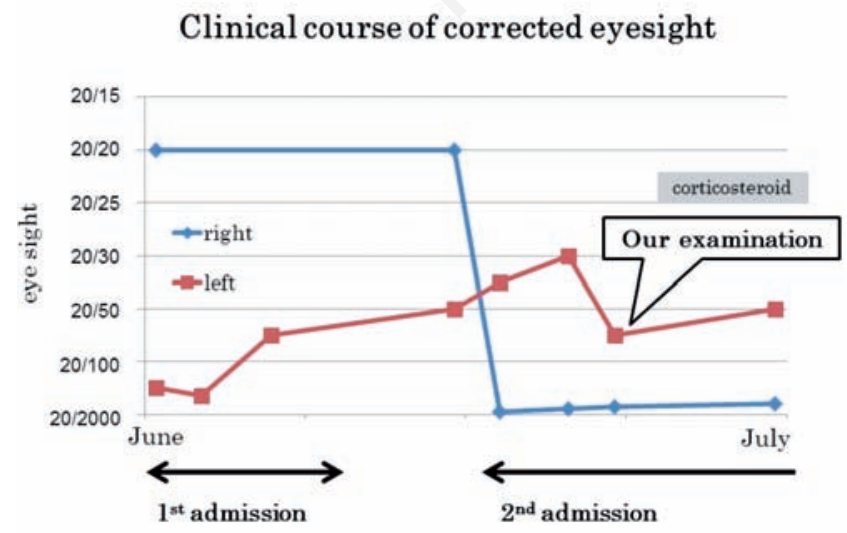

Figure 2. Clinical course of corrected eyesight. Our first examination was at the end of June; corticosteroid therapy started immediately.

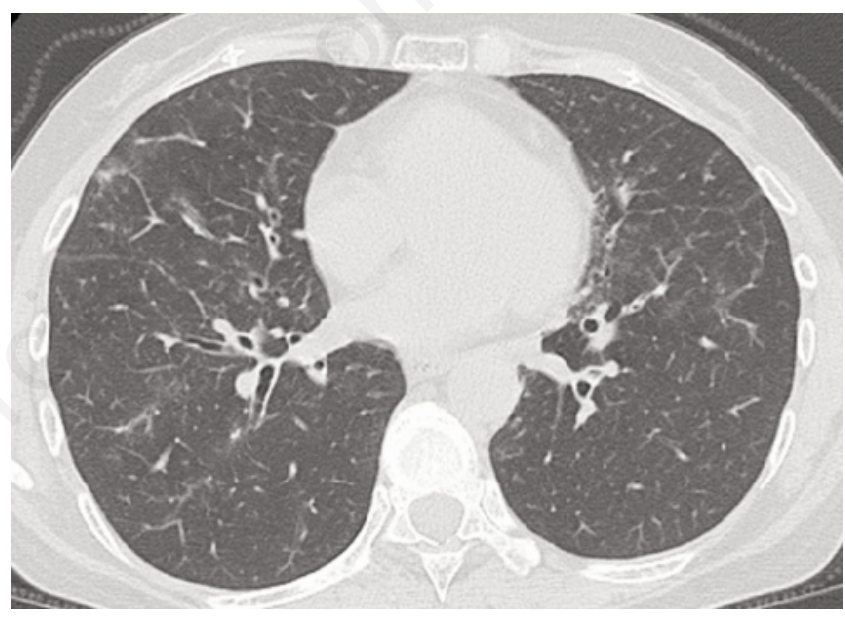

Figure 3. Chest computed tomography showing peribronchial small vesicular shadows, patchy ground-glass opacities, and thickening of the bronchus wall.

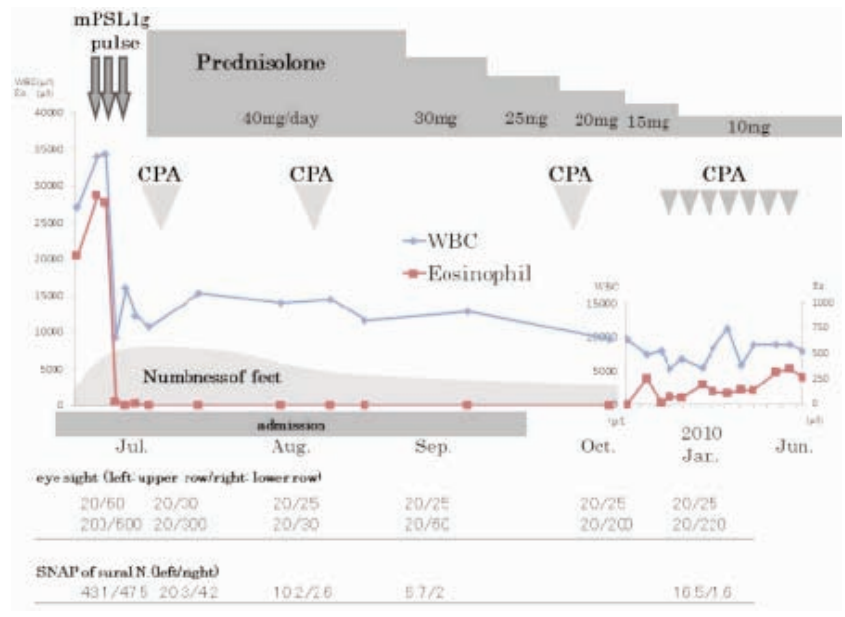

Figure 4. Summary of the clinical course. 


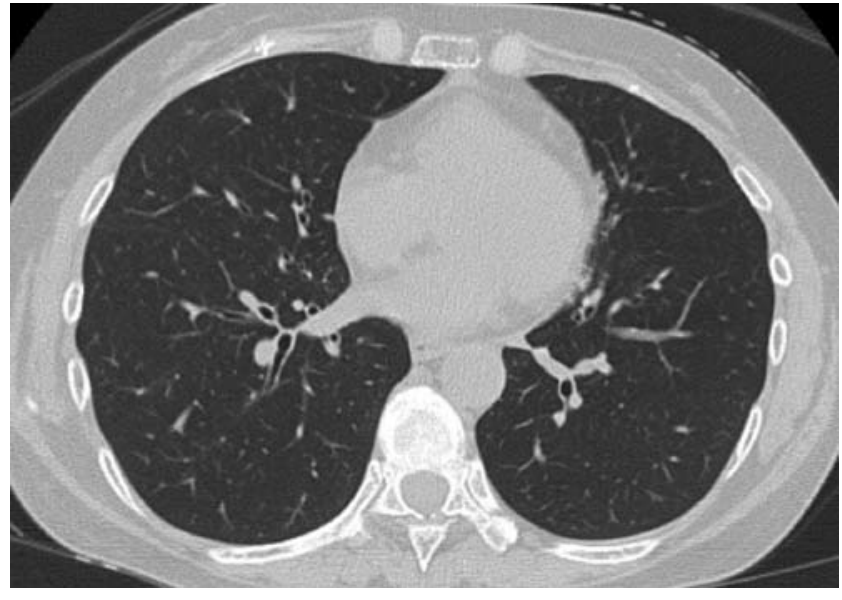

Figure 5. Chest computed tomography taken one month after the administration of treatment. All the findings shown in Figure 3 vanished except for the thickening of the bronchus wall.
Table 1. Five factor score.

\begin{tabular}{|c|c|}
\hline Five & \\
\hline Eleva & $\mathrm{ng} / \mathrm{dL})$ \\
\hline Prote & \\
\hline $\begin{array}{l}\text { Sever } \\
\text { pancr }\end{array}$ & t (perforation, bleeding, infarction, \\
\hline Cardi & \\
\hline Centr & \\
\hline FFS & 5-year mortality (\%) \\
\hline 2 & 46 \\
\hline 0 & $11.9^{\circ}$ \\
\hline
\end{tabular}

vasculitis of the retina and orbital pseudotumors consisting of granulomas are thought to cause diseases of the eye. ${ }^{1,2}$ Vasculitis, for example, is a reported cause of CRA0 ${ }^{6-8}$ central retinal venous occlusion, ${ }^{9}$ optic neuropathy ${ }^{10}$ and cilioretinal artery occlusion. ${ }^{11}$ Of the 14 patients having CSS complicated with eye lesions reported in Japan from 1995 to 2009, two had CRAO. Six cases of CSS with CRAO have been reported worldwide, whereas only two such cases have been reported in Japan (bilateral CRAO developed quickly after diagnosis in both cases). ${ }^{6,7}$ The crucial time for treating CRAO is only 100 min, and the treatment outcome worsens as the time-to-treatment lengthens. A standard therapy has yet to be established even for ophthalmologic complications. Eyeball massage and heparin administration have not been validated as effective, and results are awaited from investigations on local intra-arterial fibrinolysis with tissue plasminogen activator (t-PA) and neodymium yttrium aluminum garnet laser embolysis with tPA. ${ }^{12,13}$ The primary treatment by the ophthalmologist who first examines the eyes is extremely important, as is the sufficient flow of information and co-ordination among the ophthalmologists involved. In short, the immediate initiation of medication for the CSS is thought to be crucial for the satisfactory maintenance of eyesight and improvement of long-term quality of life (QOL). While the recommended therapy for the CSS is an initial administration of corticosteroid followed by immunosuppressant when the vasculitis is severe,,$^{14}$ there is no consensus on how to treat the eye lesions of CSS. No criterion for commencing corticosteroid or determining the initial corticosteroid dose has been proposed for such a lesion. Cyclophosphamide pulse is generally applied for CSS patients who present with more than two factors of the five factor score (FFS) (Table 1), i.e. in patients with poor prognosis. ${ }^{15}$ Camillo et al., on the other hand, achieved a $97 \%$ five-year survival rate for CSS patients by adding CPA pulse or azathioprine on top of the corticosteroid therapy, even in patients with zero FFS. ${ }^{16}$ This study revealed improvement in the 5-year survival from the previously reported $88.1 \%,{ }^{15}$ which suggests that CPA pulse is beneficial and might compensate for the risk of renal disorder or carcinogenicity associated with CPA. While the additional use of immunosuppressants is an appropriate option when corticosteroid therapy is insufficiently effective, consensus on the most beneficial treatment for eye lesions in CSS has yet to be established. In some cases corticosteroid alone has been effective, ${ }^{6}$ while concurrent immunosuppressant brings about good outcomes in steroid-resistant cases ${ }^{7,8}$ We should reconsider the option of keeping the optic sequel and lower limbs numbness tolerable through earlier initiation of CPA pulse or other immunosuppressants. Intravenous immunoglobulin treatment (IVIG) improves CSS complications such as peripheral neuropathy and cardiac disorder in cases resistant to PSL and CPA therapy. ${ }^{17}$ Intravenous immunoglobulin treatment was approved for coverage under Japan's National Health Insurance System in 2010. Intravenous immunoglobulin treatment is postulated to improve already well-established symptoms of peripheral neuropathy via mechanisms such a blood-flow improvement or direct action to the nerves, but the exact mechanisms have yet to be clarified. ${ }^{18}$ The therapies most beneficial for established disorders of the eye field or eyesight with concurrent neuropathy are still uncertain. An important question to resolve is whether IVIG improves the optic disorder.

\section{Conclusions}

Bilateral CRAO is only rarely experienced as an ophthalmic complication of CSS. The crucial time for treating CRAO is short, and the treatment outcome worsens as the time-to-treatment lengthens. The immediate initiation of medication for the CSS is thought to be crucial. While there is no consensus on how to treat the eye lesions of CSS, physicians should consider the concomitant use of corticosteroid and immunosuppressants, possibly along with IVIG, to preserve eyesight and QOL.

\section{References}

1. Nagasawa T, Yoshida M. [Clinical features of allergic granulomatous angiitis in Japan and proposed guidelines for its clinical diagnosis]. [Article in Japanese]. Nihon Naika Gakkai Zasshi 1989;78:352-6.

2. Guillevin L, Cohen P, Casassus P, et al. Churg-Strauss syndrome. Clinical study and long-term follow-up of 96 patients. Medicine 1999;78:26-37.

3. Churg J, Strauss L. Allergic granulomatosis, allergic angiitis and periarteritis nodosa Am J Pathol 1951;27:277-94.

4. Masi AT, Hunder GG, Lie JT, et al. The American College of Rheumatology 1990 criteria for the classification of ChurgStrauss syndrome (allergic granulomatous angiitis). Arthritis Rheum 1990;33:1094100.

5. Hashimoto H. [Allergic granulomatous angiitis]. In: Ministry of Health, Labour and Welfare of Japan, ed. [Countermeasures 
study project of public welfare scientific research for intractable disease, Ministry of Health, Labour, Welfare: research group on intractable vasculitis]. [Book in Japanese]. Tokyo, Japan: Ministry of Health, Labour and Welfare of Japan; 2002. pp 27-9.

6. Nakahara M, Baba T, Futagami S, et al. [Bilateral central retinal artery occlusion in a case with Churg-Strauss syndrome]. [Article in Japanese]. Ophthalmology 2005;47:869-74.

7. Udono T, Abe T, Sato H, et al. Bilateral central retinal artery occlusion in ChurgStrauss syndrome. Am J Ophthalmol 2003; 136:1181-3.

8. Matsui K, Adachi M, Nakabayashi Y, et al. [A case of Churg-Strauss syndrome with various ophthalmic symptoms]. [Article in Japanese]. Rinsho to Kenkyu 2008;85:103740.

9. Okubo E, Terashima S, Yoshida Y, et al. [A case of allergic granulomatous angiitis (limited form)]. [Article in Japanese]. Rinsho derma (Tokyo). 1995;37:1753-6.

10. Nakagawa T, Oguro H, Toyoda M, et al. [A case of allergic granulomatous angiitis (Churg-Strauss syndrome) complicated with ischemic optic neuropathy]. [Article in Japanese]. Neuro-ophthalmology Japan 2009;26:155-61.

11. Yasuda T, Shindoh H, Hatano Y, et al. [A case of allergic granulomatous angiitis (Churg-Strauss syndrome) complicated with ophthalmic symptom]. [Article in Japanese]. Rinsho Hifuka 2001;55:1027-30.

12. Inoue M. [The challenge of treatment for central retinal artery occlusion; local intraarterial fibrinolysis, Nd:YAG laser embolysis and surgical embolus removal]. [Article in Japanese]. Atarashii Ganka 2007;24:7913.

13. Hayreh SS, Zimmerman MB, Kimura A, et al. Central retinal artery occlusion. Retinal survival time. Exp Eye Res 2004;78:723-36.
14. Katshuoka N, Kawakami T, Ishiburo N, et al. [Guideline for angiitis and angiopathy]. [Article in Japanese]. Nihon Hifuka Gakkai Zasshi 2008;118:2095-187.

15. Guillevin L, Lhote F, Gayraud M, et al. Prognostic factors in polyarteritis nodosa and Churg-Strauss syndrome. A prospective study in 342 patients. Medicine 1996;75:1728.

16. Ribi C, Cohen P, Panoux C, et al. Treatment of Churg-Strauss syndrome without poorprognosis factors. Arthritis Rheum 2008;58:586-94.

17. Tsurikisawa N, Taniguchi M, Saito H, et al. Treatment of Churg-Strauss syndrome with high-dose intravenous immunoglobulin. Ann Allerg Asthma Im 2004;92:80-7.

18. Tsurikisawa N. [The treatment of ChurgStrauss syndrome for multiplex polyneuropathy]. [Article in Japanese]. Riumachika 2008;40:509-18. 